\title{
A DOUBLE INEQUALITY FOR THE RATIO OF TWO CONSECUTIVE BERNOULLI NUMBERS
}

\author{
FENG QI \\ Institute of Mathematics, Henan Polytechnic University, Jiaozuo City, Henan \\ Province, 454010, China; Department of Mathematics, College of Science, Tianjin \\ Polytechnic University, Tianjin City, 300387, China
}

\begin{abstract}
In the paper, by virtue of some properties for the Riemann zeta function, the author finds a double inequality for the ratio of two consecutive Bernoulli numbers with even indexes and analyzes the approximating accuracy of the double inequality.
\end{abstract}

\section{MAin Result}

Recall from [1, p. 804, 23.1.1] and [23, p. 3, (1.1)] that the Bernoulli numbers $B_{n}$ may be generated by

$$
\frac{z}{e^{z}-1}=\sum_{n=0}^{\infty} B_{n} \frac{z^{n}}{n !}=1-\frac{z}{2}+\sum_{k=1}^{\infty} B_{2 k} \frac{z^{2 k}}{(2 k) !}, \quad|z|<2 \pi
$$

Because the function $\frac{x}{e^{x}-1}-1+\frac{x}{2}$ is odd in $x \in \mathbb{R}$, all of the Bernoulli numbers $B_{2 k+1}$ for $k \in \mathbb{N}$ equal 0 . The first few Bernoulli numbers $B_{2 k}$ are

$$
\begin{aligned}
& B_{2}=\frac{1}{6}, \quad B_{4}=-\frac{1}{30}, \quad B_{6}=\frac{1}{42}, \quad B_{8}=-\frac{1}{30}, \quad B_{10}=\frac{5}{66}, \\
& B_{12}=-\frac{691}{2730}, \quad B_{14}=\frac{7}{6}, \quad B_{16}=-\frac{3617}{510}, \quad B_{18}=\frac{43867}{798} .
\end{aligned}
$$

It has been being a classical topic to find explicit formulas, recurrent formulas, closed expressions, and integral representations of the Bernoulli numbers $B_{2 k}$. For detailed information and recently published results, please refer to [5, 6, 7, 8, 9, 10, 11, 12, 13, 16, 20, 21, 22 and closely related references therein.

An alternative topic is to bound the Bernoulli numbers $B_{2 k}$. In [1, p. 805, 23.1.15], [4, Theorem 1.1], [23, p. 14, (1.23) and p. 23, Exercise 1.2], and the papers [3, 14, 15, some inequalities for bounding the Bernoulli numbers $B_{2 k}$ were established and collected. Most of these inequalities were refined and sharpened in [2] by

$$
\frac{2(2 k) !}{(2 \pi)^{2 k}} \frac{1}{1-2^{\alpha-2 k}} \leq\left|B_{2 k}\right| \leq \frac{2(2 k) !}{(2 \pi)^{2 k}} \frac{1}{1-2^{\beta-2 k}}, \quad k \in \mathbb{N},
$$

E-mail address: qifeng618@gmail.com, qifeng618@hotmail.com

1991 Mathematics Subject Classification. Primary 11B68; Secondary 11M06, 26D99.

Key words and phrases. inequality; ratio; Bernoulli number; Riemann zeta function; Dirichlet eta function; accuracy.

This paper was typeset using $\mathcal{A M S}_{\mathcal{S}}$-LATEX. 
where $\alpha=0$ and

$$
\beta=2+\frac{\ln \left(1-6 / \pi^{2}\right)}{\ln 2}=0.649 \ldots
$$

are the best possible in the sense that they can not be replaced respectively by any bigger and smaller constants in the double inequality (1.2).

An interesting topic is to consider the differences $\left|\overline{B_{2 k+2}}\right|-\left|B_{2 k}\right|$ and the ratio $\frac{\left|B_{2 k+2}\right|}{\left|B_{2 k}\right|}$ for $k \in \mathbb{N}$. To the best of the author's ability and knowledge, the author does not know and can not find any result on this topic.

In this paper, we are interested in considering the ratio $\frac{\left|B_{2 k+2}\right|}{\left|B_{2 k}\right|}$, which is equivalent to the difference $\ln \left|B_{2 k+2}\right|-\ln \left|B_{2 k}\right|$, for $k \in \mathbb{N}$.

Our main result is the following double inequality.

Theorem 1.1. For $k \in \mathbb{N}$, the Bernoulli numbers $B_{2 k}$ satisfy

$$
\frac{2^{2 k-1}-1}{2^{2 k+1}-1} \frac{(2 k+1)(2 k+2)}{\pi^{2}}<\frac{\left|B_{2 k+2}\right|}{\left|B_{2 k}\right|}<\frac{2^{2 k}-1}{2^{2 k+2}-1} \frac{(2 k+1)(2 k+2)}{\pi^{2}} .
$$

In Section 2, we will analyze the approximating accuracy of the double inequality 1.3. Hereafter, we will give a concise proof of the double inequality 1.3 in Section 3 Finally, in Section 4 , we give several remarks on the double inequality $(1.3$.

\section{Accuracy of the double inequality 1.3}

Before proving Theorem 1.1. we discuss the accuracy of the double inequality $(1.3)$.

For $k \in \mathbb{N}$, let

$$
B(k)=\frac{\left|B_{2 k+2}\right|}{\left|B_{2 k}\right|}, \quad L(k)=\frac{2^{2 k-1}-1}{2^{2 k+1}-1} \frac{(2 k+1)(2 k+2)}{\pi^{2}},
$$

and

$$
U(k)=\frac{2^{2 k}-1}{2^{2 k+2}-1} \frac{(2 k+1)(2 k+2)}{\pi^{2}} .
$$

In Table1, we numerically list several values of differences $B(k)-L(k)$ and $U(k)-$ $B(k)$ for $1 \leq k \leq 10$, which are computed by using the Bernoulli numbers in 1.1 and the famous software MATHEMATiCA.

TABLE 1.

\begin{tabular}{|c|c|c|c|}
\hline$k$ & 1 & 2 & 3 \\
\hline$B(k)-L(k)$ & $2.6 \ldots \times 10^{-2}$ & $2.7 \ldots \times 10^{-2}$ & $1.5 \ldots \times 10^{-2}$ \\
\hline$U(k)-B(k)$ & $4.3 \ldots \times 10^{-2}$ & $9.4 \ldots \times 10^{-3}$ & $1.8 \ldots \times 10^{-3}$ \\
\hline
\end{tabular}

\begin{tabular}{|c|c|c|c|}
\hline$k$ & 4 & 5 & 6 \\
\hline$B(k)-L(k)$ & $6.3 \ldots \times 10^{-3}$ & $2.4 \ldots \times 10^{-3}$ & $8.3 \ldots \times 10^{-4}$ \\
\hline$U(k)-B(k)$ & $3.1 \ldots \times 10^{-4}$ & $5.0 \ldots \times 10^{-5}$ & $7.7 \ldots \times 10^{-6}$ \\
\hline
\end{tabular}

\begin{tabular}{|c|c|c|c|}
\hline$k$ & 7 & 8 & 9 \\
\hline$B(k)-L(k)$ & $2.7 \ldots \times 10^{-4}$ & $8.8 \ldots \times 10^{-5}$ & $2.7 \ldots \times 10^{-5}$ \\
\hline$U(k)-B(k)$ & $1.1 \ldots \times 10^{-6}$ & $1.6 \ldots \times 10^{-7}$ & $2.2 \ldots \times 10^{-8}$ \\
\hline
\end{tabular}


These numerical values hint us the approximating accuracy of the lower and upper bounds in the double inequality 1.3 for the ratio of two consecutive Bernoulli numbers with even indexes: as $k$ becomes larger and larger, the double inequality 1.3 becomes more and more accurate.

The approximating accuracy of the double inequality 1.3 may be analytically measured by

$$
[U(k)-L(k)] \frac{4^{k-1}}{k^{2}}=\left(\frac{2^{2 k}-1}{2^{2 k+2}-1}-\frac{2^{2 k-1}-1}{2^{2 k+1}-1}\right) \frac{(2 k+1)(2 k+2)}{\pi^{2}} \frac{4^{k-1}}{k^{2}} \rightarrow \frac{3}{16 \pi^{2}}
$$

as $k \rightarrow \infty$. In other words, as $k \rightarrow \infty$, we have $U(k)-L(k) \sim \frac{3 k^{2}}{16 \pi^{2} 4^{k-1}}$.

For comparison, we now consider the difference between the upper and lower bounds of the double inequality 1.2 . This difference equals

$$
\frac{2(2 k) !}{(2 \pi)^{2 k}}\left[\frac{1}{1-2^{\beta-2 k}}-\frac{1}{1-2^{\alpha-2 k}}\right]=2\left(2^{\beta}-1\right) \frac{2^{2 k}}{2^{2 k}-2^{\beta}} \frac{(2 k) !}{(2 \pi)^{k}\left(2^{2 k}-1\right)}
$$

which diverges to $\infty$ as $k \rightarrow \infty$.

In a word, the double inequality 1.3 provides a better manner than 1.2 to estimate the Bernoulli numbers $B_{2 k}$ for $k \in \mathbb{N}$.

\section{Proof of Theorem 1.1}

We are now in a position to prove the double inequality 1.3 .

Recall from [1, p. 807, 23.2.1] and [23, p. 57] that the Riemann zeta function $\zeta$ may be defined by the series $\zeta(z)=\sum_{n=1}^{\infty} \frac{1}{n^{z}}$ under the condition $\Re(z)>1$, and by analytic continuation elsewhere.

In $[23$, p. $5,(1.14)]$, it was listed that

$$
B_{2 k}=\frac{(-1)^{k+1} 2(2 k) !}{(2 \pi)^{2 k}} \zeta(2 k), \quad k \in \mathbb{N} .
$$

Then

$$
\frac{\left|B_{2 k+2}\right|}{\left|B_{2 k}\right|}=\frac{(2 k+1)(2 k+2)}{\pi^{2}} \frac{1}{4} \frac{\zeta(2 k+2)}{\zeta(2 k)}, \quad k \in \mathbb{N} .
$$

Hence, to prove the right-hand side of the inequality 1.3 , it is sufficient to verify

$$
\frac{1}{4} \frac{\zeta(2 k+2)}{\zeta(2 k)}<\frac{2^{2 k}-1}{2^{2 k+2}-1}
$$

which may be rearranged as

$$
\left(1-\frac{1}{2^{2 k+2}}\right) \zeta(2 k+2)<\left(1-\frac{1}{2^{2 k}}\right) \zeta(2 k), \quad k \in \mathbb{N} .
$$

This inequality is a special case of [4, Lemma 2.1] and [25, Lemma 2.1] which may be slightly modified as follows: the sequence

$$
\left(1-\frac{1}{2^{k}}\right) \zeta(k)=\zeta(k)-\sum_{m=1}^{\infty} \frac{1}{(2 m)^{k}}=\sum_{m=1}^{\infty} \frac{1}{(2 m-1)^{k}}, \quad k \geq 2
$$

is decreasing in $k$. The right-hand side of the inequality $\sqrt{1.3}$ is thus proved.

In [24, p. 4, Corollary 1], it was derived that the Dirichlet eta function

$$
\eta(x)=\left(1-\frac{1}{2^{x-1}}\right) \zeta(x)
$$


is strictly logarithmically concave on $(0, \infty)$. This implies that the logarithmic derivative

$$
[\ln \eta(x)]^{\prime}=\frac{\eta^{\prime}(x)}{\eta(x)}=\frac{\zeta^{\prime}(x)}{\zeta(x)}-\frac{\ln 2}{2^{x-1}-1}
$$

is strictly decreasing on $(0, \infty)$. It is clear that

$$
\lim _{x \rightarrow \infty} \frac{\eta^{\prime}(x)}{\eta(x)}=\lim _{x \rightarrow \infty} \frac{\zeta^{\prime}(x)}{\zeta(x)}=0 .
$$

Consequently, it follows that $\frac{\eta^{\prime}(x)}{\eta(x)}>0$, which implies that $\eta^{\prime}(x)>0$ and $\eta(x)$ is strictly increasing, on $(0, \infty)$. As a result, we have

$$
\left(1-\frac{1}{2^{2 k+1}}\right) \zeta(2 k+2)>\left(1-\frac{1}{2^{2 k-1}}\right) \zeta(2 k)
$$

which may be rearranged as

$$
\frac{1}{4} \frac{\zeta(2 k+2)}{\zeta(2 k)}>\frac{2^{2 k-1}-1}{2^{2 k+1}-1}
$$

for $k \in \mathbb{N}$. Combining this with (3.1) brings about the left-hand side of the inequality 1.3 . The proof of Theorem 1.1 is complete.

\section{REMARKS}

Finally, we give several remarks on the double inequality 1.3 .

Remark 4.1. Motivated by the double inequality $(1.3)$ and by the fact that the function $\frac{2^{2 k+x}-1}{2^{2 k+2+x}-1}$ is strictly increasing in $x \neq-2(k+1)$ for all $k \in \mathbb{N}$, we naturally pose a question: what are the ranges of $\alpha$ and $\beta$ such that the double inequality

$$
\frac{2^{2 k+\beta}-1}{2^{2 k+2+\beta}-1} \frac{(2 k+1)(2 k+2)}{\pi^{2}}<\frac{\left|B_{2 k+2}\right|}{\left|B_{2 k}\right|}<\frac{2^{2 k+\alpha}-1}{2^{2 k+2+\alpha}-1} \frac{(2 k+1)(2 k+2)}{\pi^{2}}
$$

is valid for $n \in \mathbb{N}$ ?

By (3.1), the double inequality (4.1) can be rewritten as

$$
\frac{2^{2 k+\beta}-1}{2^{2 k+2+\beta}-1}<\frac{1}{4} \frac{\zeta(2 k+2)}{\zeta(2 k)}<\frac{2^{2 k+\alpha}-1}{2^{2 k+2+\alpha}-1}
$$

which can be further reformulated as

$$
\left(1-\frac{1}{2^{2 k+\beta}}\right) \zeta(2 k)<\left(1-\frac{1}{2^{2 k+2+\beta}}\right) \zeta(2 k+2)
$$

and

$$
\left(1-\frac{1}{2^{2 k+2+\alpha}}\right) \zeta(2 k+2)<\left(1-\frac{1}{2^{2 k+\alpha}}\right) \zeta(2 k) .
$$

Let

Then

$$
S_{\theta}(x) \triangleq\left(1-\frac{1}{2^{2 x+\theta}}\right) \zeta(2 x), \quad x \in[1, \infty), \quad \theta \in \mathbb{R} .
$$

$$
S_{\theta}^{\prime}(x)=\frac{1}{2^{2 x+\theta-1}}\left[\left(2^{2 x+\theta}-1\right) \zeta^{\prime}(2 x)+(\ln 2) \zeta(2 x)\right] .
$$

In order that the function $S_{\theta}(x)$ is strictly increasing (or strictly decreasing, respectively) on $[1, \infty)$, it is necessary and sufficient that

$$
\left(2^{2 x+\theta}-1\right) \zeta^{\prime}(2 x)+(\ln 2) \zeta(2 x) \gtreqless 0
$$


on $[1, \infty)$, which can be rearranged as

$$
2^{\theta} \lesseqgtr\left[1-\frac{(\ln 2) \zeta(2 x)}{\zeta^{\prime}(2 x)}\right] \frac{1}{2^{2 x}} \rightarrow \begin{cases}1, & x \rightarrow \infty \\ \frac{1}{4}-\frac{\pi^{2} \ln 2}{24 \zeta^{\prime}(2)}=0.55 \ldots, & x \rightarrow 1^{+} .\end{cases}
$$

Consequently, in order that the function $S_{\theta}(x)$ for $x \in[1, \infty)$ and the sequence $S_{\theta}(k)$ with $k \in \mathbb{N}$ are strictly increasing (or strictly decreasing, respectively), it is necessary that $\theta \geq 0$ (or

$$
\theta \leq \frac{\ln \left[\frac{1}{4}-\frac{\pi^{2} \ln 2}{24 \zeta^{\prime}(2)}\right]}{\ln 2}=-0.85 \ldots
$$

respectively).

The double inequality 4.2 can also be reformulated as

$$
2^{2+\beta}<\frac{1}{2^{2 k}} \frac{4-\zeta(2 k+2) / \zeta(2 k)}{1-\zeta(2 k+2) / \zeta(2 k)} \quad \text { and } \quad 2^{2+\alpha}>\frac{1}{2^{2 k}} \frac{4-\zeta(2 k+2) / \zeta(2 k)}{1-\zeta(2 k+2) / \zeta(2 k)}
$$

Since

$$
\frac{1}{2^{2 k}} \frac{4-\zeta(2 k+2) / \zeta(2 k)}{1-\zeta(2 k+2) / \zeta(2 k)} \rightarrow \begin{cases}\frac{\pi^{2}-60}{4\left(\pi^{2}-15\right)}=2.44 \ldots, & k \rightarrow 1 \\ 4, & k \rightarrow \infty\end{cases}
$$

It follows that the necessary conditions are $\alpha \geq 0$ and

$$
\beta \leq \frac{\ln \frac{\pi^{2}-60}{\pi^{2}-15}}{\ln 2}-4=-0.711 \ldots
$$

This implies that the right-hand side inequality in 1.3 is sharp, but the left-hand side inequality in 1.3 perhaps may be improved.

In conclusion, we guess that the double inequality 4.1 is valid if and only if $\alpha \geq 0$ and

$$
\beta \leq \frac{\ln \left[\frac{1}{4}-\frac{\pi^{2} \ln 2}{24 \zeta^{\prime}(2)}\right]}{\ln 2}=-0.85 \ldots
$$

In order to verify this guess, perhaps we need the guess posed in Remark 4.2 below.

Remark 4.2. Since

$$
\lim _{x \rightarrow 1^{+}}\left\{\left[1-(\ln 2) \frac{\zeta(x)}{\zeta^{\prime}(x)}\right] \frac{1}{2^{x}}\right\}=\frac{1}{2} \quad \text { and } \quad \lim _{x \rightarrow \infty}\left\{\left[1-(\ln 2) \frac{\zeta(x)}{\zeta^{\prime}(x)}\right] \frac{1}{2^{x}}\right\}=1,
$$

we guess that the function

$$
\left(1-\frac{1}{2^{x+\theta}}\right) \zeta(x), \quad x \in(1, \infty)
$$

is strictly increasing (or strictly decreasing, respectively) if and only if $\theta \leq-1$ (or $\theta \geq 0$, respectively).

Remark 4.3. This paper is a corrected and simplified version of the preprints [17, 18, 19. 
Acknowledgements. The author thanks Dr. Dongkyu Lim, a Korean mathematician and a postdoctoral of the Nankai University in China, for his carefully reading and corrections to the original version of this paper.

This paper was reported on the 2015 Conference and Special Seminar of the Jangjeon Mathematical Society held at the Hannam University and Kyungpook National University in Daejeon City and Daegu City between 25-31 May 2015 and the 7th Conference of the Society for Mathematical Inequalities and Applications in China (SMIAChina) held at Hunan City University at Yiyang City in China between 8-9 August 2015.

\section{REFERENCES}

[1] M. Abramowitz and I. A. Stegun (Eds), Handbook of Mathematical Functions with Formulas, Graphs, and Mathematical Tables, National Bureau of Standards, Applied Mathematics Series 55, 10th printing, Washington, 1972.

[2] H. Alzer, Sharp bounds for the Bernoulli numbers, Arch. Math. (Basel) 74 (2000), no. 3, 207-211; Available online at http://dx.doi.org/10.1007/s000130050432

[3] C. D'Aniello, On some inequalities for the Bernoulli numbers, Rend. Circ. Mat. Palermo (2) 43 (1994), no. 3, 329-332; Available online at http://dx.doi.org/10.1007/BF02844246

[4] H.-F. Ge, New sharp bounds for the Bernoulli numbers and refinement of Becker-Stark inequalities, J. Appl. Math. 2012, Art. ID 137507, 7 pages; Available online at http: //dx.doi.org/10.1155/2012/137507

[5] H. W. Gould, Explicit formulas for Bernoulli numbers, Amer. Math. Monthly 79 (1972), no. 1, 44-51; Available online at http://dx.doi.org/10.2307/2978125

[6] R. L. Graham, D. E. Knuth, and O. Patashnik, Concrete Mathematics-A Foundation for Computer Science, Addison-Wesley Publishing Company, Advanced Book Program, Reading, MA, 1989

[7] R. L. Graham, D. E. Knuth, and O. Patashnik, Concrete Mathematics-A Foundation for Computer Science, 2nd ed., Addison-Wesley Publishing Company, Reading, MA, 1994.

[8] B.-N. Guo and F. Qi, A new explicit formula for the Bernoulli and Genocchi numbers in terms of the Stirling numbers, Glob. J. Math. Anal. 3 (2015), no. 1, 33-36; Available online at http://dx.doi.org/10.14419/gjma.v3i1.4168

[9] B.-N. Guo and F. Qi, An explicit formula for Bernoulli numbers in terms of Stirling numbers of the second kind, J. Anal. Number Theory 3 (2015), no. 1, 27-30; Available online at http://dx.doi.org/10.12785/jant/030105

[10] B.-N. Guo and F. Qi, Some identities and an explicit formula for Bernoulli and Stirling numbers, J. Comput. Appl. Math. 255 (2014), 568-579; Available online at http://dx.doi. org/10.1016/j.cam.2013.06.020

[11] S.-L. Guo and F. Qi, Recursion formulae for $\sum_{m=1}^{n} m^{k}$, Z. Anal. Anwendungen 18 (1999), no. 4, 1123-1130; Available online at http://dx.doi.org/10.4171/ZAA/933

[12] J. Higgins, Double series for the Bernoulli and Euler numbers, J. London Math. Soc. 2nd Ser. 2 (1970), 722-726; Available online at http://dx.doi.org/10.1112/jlms/2.Part_4.722

[13] S. Jeong, M.-S. Kim, and J.-W. Son, On explicit formulae for Bernoulli numbers and their counterparts in positive characteristic, J. Number Theory 113 (2005), no. 1, 53-68; Available online at http://dx.doi.org/10.1016/j.jnt.2004.08.013

[14] A. Laforgia, Inequalities for Bernoulli and Euler numbers, Boll. Un. Mat. Ital. A (5) 17 (1980), no. 1, 98-101.

[15] D. J. Leeming, The real zeros of the Bernoulli polynomials, J. Approx. Theory 58 (1989), no. 2, 124-150; Available online at http://dx.doi.org/10.1016/0021-9045(89)90016-6.

[16] B. F. Logan, Polynomials related to the Stirling numbers, AT\&T Bell Laboratories internal technical memorandum, August 10, 1987.

[17] F. Qi, A double inequality for ratios of the Bernoulli numbers, ResearchGate Dataset (2015), available online at http://dx.doi.org/10.13140/RG.2.1.3461.2641

[18] F. Qi, A double inequality for ratios of Bernoulli numbers, ResearchGate Dataset (2014), available online at http://dx.doi.org/10.13140/2.1.2367.2962

[19] F. Qi, A double inequality for ratios of Bernoulli numbers, RGMIA Research Report Collection 17 (2014), Article 103, 4 pages; Available online at http://rgmia.org/v17.php 
[20] F. Qi, Derivatives of tangent function and tangent numbers, Appl. Math. Comput. 268 (2015), 844-858; Available online at http://dx.doi.org/10.1016/j.amc.2015.06.123

[21] F. Qi and B.-N. Guo, Alternative proofs of a formula for Bernoulli numbers in terms of Stirling numbers, Analysis (Berlin) 34 (2014), no. 3, 311-317; Available online at http: //dx.doi.org/10.1515/anly-2014-0003

[22] S. Shirai and K.-I. Sato, Some identities involving Bernoulli and Stirling numbers, J. Number Theory 90 (2001), no. 1, 130-142; Available online at http://dx.doi.org/10.1006/jnth. 2001.2659

[23] N. M. Temme, Special Functions: An Introduction to Classical Functions of Mathematical Physics, A Wiley-Interscience Publication, John Wiley \& Sons, Inc., New York, 1996; Available online at http://dx.doi.org/10.1002/9781118032572.

[24] K. C. Wang, The logarithmic concavity of $\left(1-2^{1-r}\right) \zeta(r)$, J. Changsha Comm. Univ. 14 (1998), no. 2, 1-5. (Chinese)

[25] L. Zhu and J.-K. Hua, Sharpening the Becker-Stark inequalities, J. Inequal. Appl. 2010 (2010), Art. ID 931275, 4 pages; Available online at http://dx.doi.org/10.1155/2010/ 931275

$U R L:$ https : //qifeng618.wordpress.com 